\title{
Preoperative Aspirin Use and Outcomes in Off-pump Coronary Artery Bypass Grafting Surgery
}

\author{
Md. Rezaul Karim ${ }^{1}$, Tawfiq Ahmed ${ }^{2}$, Rownak Khurshid ${ }^{3}$, Shahriar Moinuddin ${ }^{3}$, Md. Kamrul Hasan ${ }^{4}$
}

\begin{abstract}
:
Introduction: Aspirin, the most widely used platelet function inhibitor extremely effective at blocking the production of thromboxane in platelets, rendering the platelets incapable of functioning normally, and thus preventing thrombosis. The practice of empirically discontinuing aspirin preoperatively should be abandoned because evidence strongly supports continued use of aspirin in patients for secondary prevention of CAD, CVD, or PVD when undergoing surgery.
\end{abstract}

Methods and Materials: This Observational study was conducted at Department of Cardiac Surgery, NICVD, Dhaka, who underwent off pump CABG (OPCAB), divided in two groups, Group A: 24 patients who stopped and Group B: 24 patients who are continuing aspirin throughout the perioperative period. Post operative blood loss, requirement of blood transfusion, post-operative MI, ICU stay, Total hospital stay (days) and early post-operative complication (Stroke, New arrhythmia in ECG, 30 days mortality) were recorded and included in the study.

Results: The key finding of the present study is that preoperatively continued aspirin use was not associated with increased risk of post-operative blood loss, blood transfusion requirements and need for re exploration after OPCAB.

Conclusions: Preoperative aspirin therapy should be continued till off-pump CABG without interruption.

Key words: Aspirin, Coronary Artery Bypass, Off-pump.
Introduction:

The platelet is integral to the initiation of thrombosis. Platelets are anucleate cells produced daily from bone marrow megakaryocytes and have a lifespan of 8 to 10 days. ${ }^{1}$ Drugs that affect platelet function are a fundamental part of primary and secondary management of atherosclerotic thrombotic disease.

Aspirin, the most widely used platelet function inhibitor mediates its effects through the arachidonic acid (AA) -

1. Assistant Professor, Department of Cardiac Surgery, National Institute of Cardiovascular Diseases, Dhaka, Bangladesh.

2. Assistant Professor, Department of Cardiac Surgery, Sir Salimullah Medical College, Dhaka, Bangladesh.

3. Assistant Registrar, Department of cardiac Surgery, National Institute of Cardiovascular Diseases, Dhaka, Bangladesh.

4. Professor and Head, Department of cardiac Surgery, National Institute of Cardiovascular Diseases, Dhaka, Bangladesh.

Address of Correspondence: Dr. Md. Rezaul Karim, Assistant Professor, Department of Cardiac Surgery, National Institute of Cardiovascular Diseases, Sher-e-Bangla Nagar, Dhaka, Bangladesh. Mobile: +8801736126852, E-mail-drrezanicvd@gmail.com thromboxane $A_{2}\left(T x A_{2}\right)$ pathway. The conversion of $A A$ to prostaglandin occurs throughout the body and is catalyzed by the enzyme cyclooxygenase (COX). There are 2 isoforms of COX, termed COX-1 and COX-2. Aspirin irreversibly inactivates COX through acetylation of the amino acid serine, with a 170-fold affinity for COX-1 over COX-2. By inactivating COX-1, aspirin renders the platelet incapable of synthesizing prostaglandin $\mathrm{H}_{2}$. Consequently, aspirin is extremely effective at blocking the production of thromboxane in platelets, rendering the platelets incapable of functioning normally, and thus preventing thrombosis and the damaging cardiovascular events that may result. ${ }^{2}$

Aspirin may further diminish the risk of cardiovascular disease through its ability to decrease inflammation by blocking C-reactive protein, although this benefit probably only occur at higher doses than those used clinically. ${ }^{3}$

Surgical intervention induces a catecholamine surge which in turn augments inflammation and platelet reactivity, the 
link between peri-operative stressors and inadvertent thrombosis becomes more clear. ${ }^{4}$ Moreover, it has been demonstrated that this catecholamine-induced platelet reactivity is only partly counteracted by aspirin therapy. ${ }^{5}$

A growing body of evidence supports a platelet rebound phenomenon in the setting of acute aspirin withdrawal. This rebound period is characterized by increased thromboxane production, decreased fibrinolysis, and a resultant clinical prothrombotic state. ${ }^{6}$

On the basis of the available evidence, the practice of empirically discontinuing aspirin preoperatively should be abandoned. The evidence strongly supports continued use of aspirin in patients on it for secondary prevention of CAD, CVD, or PVD when undergoing surgery. Routine discontinuation of aspirin 7 to 10 days preoperatively is not only unjustified but likely significantly compounds patient's thromboembolic risk because of the described aspirin withdrawal syndrome that occurs contemporaneously during this time interval. For an at-risk patient, the hypercoagulable state engendered by the surgical procedure compounded by the aspirin withdrawal syndrome creates an ideal scenario for a major cardiac or vascular thromboembolic complication. $^{7}$

In 2010, the European Society of Cardiology and the European Association for Cardio-Thoracic Surgery recommended that preoperative aspirin should not be stopped $^{8}$ and in 2011, the American College of Cardiology Foundation and the American Heart Association guideline for coronary artery bypass grafting (CABG) recommended that aspirin (100 to $325 \mathrm{mg}$ daily) should be administered to CABG patients preoperatively. ${ }^{9}$ Some well-conducted studies have shown that aspirin use before coronary artery bypass procedures is safe without an associated increase in hemorrhage-related risks and could reduce in-hospital mortality. ${ }^{10}$

The relationship of aspirin to graft patency after conventional CABG has been studied extensively at both the clinical and the pathophysiologic levels, and it is generally accepted as established fact that aspirin therapy increases graft patency. ${ }^{11}$

Tuman and his colleagues compared perioperative use of aspirin versus placebo in patients undergoing coronary artery bypass graft (CABG). They found no significant differences in postoperative hematocrit, mediastinal bleeding, transfusion requirements and need for re exploration. ${ }^{12}$ Srinivasan and his colleagues retrospectively examined and found similar result. ${ }^{13}$

However, few published data have evaluated the effect of preoperative continuation of aspirin therapy on perioperative graft patency after off-pump coronary artery bypass (OPCAB). OPCAB has offered a promising alternative strategy that had the potential to decrease perioperative morbidity, mortality, and cost by eliminating cardiopulmonary bypass, but there is growing concern that OPCAB is associated with reduced graft patency. Platelet inhibition with aspirin has been shown to reduce the rates of acute and sub-acute bypass graft occlusion.

To evaluate the potential effects of preoperative continuation of aspirin therapy in patients undergoing OPCAB, we performed this study.

\section{Materials and Methods:}

This observational study was conducted at Department of Cardiac Surgery, NICVD, Dhaka from July 2015 to July 2016. Total 48 patients who will undergo OPCAB were selected for the study divided in two groups, Group A: 24 patients who stopped Aspirin 5 days before operation and Group B: 24 patients who are continuing aspirin throughout the perioperative period. Patients were excluded from the study if there is history of Ml within 6 weeks, hepatic dysfunction, renal dysfunction, Redo CABG, combined CABG and conversion CABG. Patient taking anticoagulant, patient with history of stenting and patient requiring end-arterectomy were also excluded from the study. Detailed history of each patient under study and important and relevant findings on thorough physical examinations and investigations were recorded. A standard anesthetic and heparin protocol used throughout the study. All patients was routinely given aspirin $75 \mathrm{mg}$ daily postoperatively, with the first dose of aspirin (75 $\mathrm{mg}$ ) being administrated on the operative day $6 \mathrm{hrs}$ after operation. Following the surgical procedure, all the patients were brought to the Cardiovascular ICU where they were monitored until extubation and stabilization of respiratory and hemodynamic status. Then the patients were transferred to the HDU from there to the ward for routine care. The patients were discharged from the ward and advised for subsequent follow up after 1 month and 3 month.

Post operative mediastinal bleeding, requirement of blood transfusion, need for re exploration, post-operative MI, ICU stay, Total hospital stay (days) and early post-operative complication (Stroke, New arrhythmia in ECG, 30 days mortality) were recorded and included in the study. Statistical analysis of the results was done by SPSS. The results were presented in Tables, Figures and Diagrams etc.

\section{Results:}

The present study is intended to assess short term outcome of perioperative continuation of aspirin in patients undergoing OPCAB. A total of 48 (forty eight) patients were recruited for the purpose of the study. The recruited patients were 
assigned into two groups according to their preoperative aspirin use. Group A patients (without aspirin, $n=24$ ) consists of patients stopped aspirin 5 days before operation. Group $B$ patients (with aspirin, $n=24$ ) consists of the patients who have continued aspirin. Postoperative mediastinal bleeding, required amount of blood transfusion, peri and post-operative other parameters were measured in both the groups. The findings of the study obtained from data analyses are presented below.

Table-I

Pre-operative variable

\begin{tabular}{lccc}
\hline & Group I $(\mathrm{n}=24)$ & Group II $(\mathrm{n}=24)$ & $\mathrm{p}$-value \\
\hline Age (years) & $52.96 \pm 10.02$ & $52.67 \pm 8.28$ & .913 \\
Male & $20(83.4 \%)$ & $22(91.74 \%)$ & .383 \\
Female & $4(16.66 \%)$ & $2(9.34 \%)$ & \\
Hypertension & $13(54.16 \%)$ & $14(58.33 \%)$ & .77 \\
Diabetes mellitus & $10(41.17 \%)$ & $10(41.17 \%)$ & 1.00 \\
Dyslipidemia & $19(79.16 \%)$ & $17(70.83 \%)$ & .44 \\
Haematocrit $(\%)$ & $38.83 \pm 3.49$ & $37.08 \pm 8.68$ & .364 \\
Bleeding time (min) & $4.45 \pm 1.10$ & $4.43 \pm 1.10$ & .803 \\
Clotting time (min) & $5.6 \pm 0.88$ & $5.57 \pm .87$ & .916 \\
\hline
\end{tabular}

Table I shows that the age of the patients undergoing OPCAB ranges from 35 years to 65 years. But most of the patients were in the range of 46 to 60 years (Group A58.38\%, Group B $62.52 \%)$. Moreover there was no statistical significant difference between the groups in terms of age $(p>0.05)$. There was no significant difference between the groups in terms of sex. But shows that there is a male dominance among the patients group $\mathrm{A}(83.3 \%$ against $16.66 \%)$ and group $\mathrm{B}(91.74 \%$ against $9.34 \%)$. Patients in both the groups were statistically identical according to the base line clinical characteristics. Prevalence of diabetes mellitus were almost similar in both group (Group A $41.17 \%$ vs Group B $41.17 \%$ ), Regarding hypertension, dyslipidemia Group Aand Group B were almost similar (54.16 $\%$ to $58.33 \%, p=1.00 ; 79.16 \%$ to $70.83 \%, p=.44$ ).

There was no statistically significant difference among the patients Haematocrit, Bleeding

Time and Clotting time in minutes $(38.83 \pm 3.49$ vs $37.08 \pm 8.68 ; p=.364,4.45 \pm 1.10$ vs $4.43 \pm 1.10, p=.803$; $5.6 \pm 0.88$ vs $5.57 \pm .87, p=.916$ respectively ).

In pre-operative echocardiographic study left ventricular ejection fraction were studied in all patients. LVEF was divided into two groups. One was more than $50 \%$ and other was less than $50 \%$. In group A more than $18(75 \%)$ of the patient had LVEF $<50$ and $6(25 \%)$ had LVEF $>50$. In GroupB $17(70.8 \%)$ patient had LVEF $<50$ and 7 (29.2\%) patient had LVEF $>50$. But there was no significant difference in LVEF among the groups.
Pre-operative angiogram shows that there was also no significant difference between the two groups, most of the patients have triple vessel disease, $16(66.66 \%)$ in Group A and 15 (62.5\%) in Group - B.

There was no significant difference between the two groups with regard to operative time, most of the operations perform 4.5 hrs to 6.5 hrs, Group A and Group B respectively $5.60 \pm 0.91 \& 5.40 \pm 0.71$. Difference between the two groups with regard to number of graft also not significant but most of the patients given three grafts (Group A $75.06 \%$ vs Group B $84.33 \%$ respectively). There is no significant difference between 2 groups with regards to activated clotting time, most of the reversal done at $100-120$ seconds, $91.6 \%$ and $87.5 \%$ in Group A and Group - B respectively.

Table-II

Post-operative bleeding and blood transfusion

\begin{tabular}{lccc}
\hline & Group I & Group II & $\mathrm{P}$-value \\
\hline $\begin{array}{l}\text { Up to removal } \\
\text { of DT }(\mathrm{ml})\end{array}$ & $643.33 \pm 36.67$ & $648.33 \pm 37.61$ & .643 \\
$\begin{array}{l}\text { Blood Transfusion } \\
\begin{array}{l}\text { Reoperation for } \\
\text { bleeding }\end{array}\end{array}$ & $1.16 \pm 0.38$ & $1.12 \pm .33$ & .69 \\
\hline
\end{tabular}

Average amount of bleeding measured at the end of 24 hours and then up to removal of drain tubes. There was no significant difference between the two groups with regard to bleeding. At 24 hours $371.67 \pm 72.15 \mathrm{ml}$ blood loss measured in Group A and $370.83 \pm 71.98 \mathrm{ml}$ in Group -B. During removal of drain tube total $643.33 \pm 36.67 \mathrm{ml}$ and $648.33 \pm 37.61 \mathrm{ml}$ in Group-A and Group-B respectively. Shows there was no significant difference between the two groups with regard to blood transfusion. Most of patients needed 1 unit blood transfusion in both group (Group A 83.4\% vs Group B $87.57 \%$ ). No patient required reoperation for bleeding. (Table II).

Table-III

Post-operative ECG changes

\begin{tabular}{lccc}
\hline & Group I & Group II & P-value \\
\hline Normal & $22(91.74 \%)$ & $23(95.91 \%)$ & .221 \\
Q Wave & $2(8.34 \%)$ & $1(4.17 \%)$ & \\
ST changes & $2(8.34 \%)$ & $1(4.17 \%)$ & \\
New arrhythmia & $6(25.022 \%)$ & $5(20.85 \%)$ & .731 \\
\hline
\end{tabular}

Post-operative ECG changes shows there was no significant difference between the two groups. Most of patients have normal ECG Group A 91.74\% vs Group B 95.91\% respectively. ST elevation appears in 2 (8.33\%) patients in Group A and 1 (4.16\%) patients in Group B. Q wave appears in 2 (8.33\%) 
patients in Group Aand 1 (4.16\%) patients in Group B. There was no significant difference between the two groups with regard to new arrhythmia (Group A $25.02 \%$ \& Group B $20.85 \%$ ). Post-operative trop I level difference was also nonsignificant between 2 groups. (Table III)

There was no significant difference between the two groups with regard to ICU stay. But most of the patients stayed 4 days, Group A $83.4 \%$ vs Group B $79.23 \%$ respectively.

Hospital stay was also not significant between the two groups. But most of patients released less than 10 days (Group A $70.89 \%$ vs Group B 75.06\%).

Table IV

Follow-up variable

\begin{tabular}{lccc}
\hline & $\begin{array}{c}\text { GroupA } \\
(\mathrm{n}=24)\end{array}$ & $\begin{array}{c}\text { Group B } \\
(\mathrm{n}=24)\end{array}$ & p-value \\
\hline 30 day mortality & $1(4.16 \%)$ & 0 & .074 \\
Ejection Fraction & & & \\
1 month & $46.66 \pm 3.97$ & $46.04 \pm 4.65$ & .633 \\
3 month & $55.9 \pm 3.7$ & $54.79 \pm 4.77$ & .392 \\
Postoperative complication & & & \\
Thromboembolism & $\mathrm{Nil}$ & $\mathrm{Nil}$ & $\mathrm{N} / \mathrm{A}$ \\
DVT & $\mathrm{Nil}$ & $\mathrm{Nil}$ & $\mathrm{N} / \mathrm{A}$ \\
CVA & $\mathrm{Nil}$ & $\mathrm{Nil}$ & $\mathrm{N} / \mathrm{A}$ \\
\hline & & &
\end{tabular}

There was no significant difference between the two groups with regard to 30 days mortality. 1 patients died within 30 days in Group-A. No mortality in Group B aspirin users. There was no significant difference between the two groups with regard to ejection fraction 1 month after discharge. There was no incidence of thromboembolism, DVT or CVAin either group seen upto 3 months of followup. ( Table IV )

\section{Discussion:}

The effect of preoperative aspirin administration remains unclear in off-pump CABG. With an increasing volume of Off-pump CABG performed in Asian countries which accounts for at least $60 \%$ of all the CABG. In consideration of the significant differences in term of postoperative coagulation system between off-pump CABG and on-pump $C A B G$, it is essential to evaluate the preoperatively continued aspirin use until surgery in OPCAB patients.

The age of the patients undergoing OPCAB ranges from 35 years to 65 years. But most of the patients were in the range of 46 to 60 years (Group A $58.38 \%$, Group B $62.52 \%$ ). Mean \pm SD age of the study subjects was $52.96 \pm 10.02$ and $52.67 \pm 8.28$ years in Group-A and Group-B respectively which was not statistically significant $(p>0.05)$. Hossain and his colleagues reported the mean age of patients undergoing OPCAB surgery to be $53.80 \pm 8.57$ years which are similar to this study.
Male sex predominates among the study subjects $(91.67 \%$ male against $9.33 \%$ female). Both the groups were comparable in respect of sex. Hossain S (2013) also reported a male preponderance in patients undergoing OPCAB surgery.

Baseline clinical characteristics in the study in both groups subjects are similar. Most of the patients were hypertensive, diabetic, dyslipidemic ( $54.16 \%$ vs $58.33 \%, 41.17 \%$ vs $41.17 \%$ and $79.16 \%$ vs $70.83 \%$ respectively).

Xiao and his colleagues reported $35.3 \%$ and $36.8 \%$ prevalence of DM respectively in Group A and Group B, prevalence of preoperative hypertension were $65.5 \%$ and $68.4 \%$ in Group A and Group B respectively patients undergoing $O P C A B$ surgery. Our study subjects were comparable in terms of preoperative patient characteristics and cardiopulmonary functional status. So postoperative clinical outcomes were not influenced by these factors.

Preoperative coagulation profiles of Group A and Group B patients were statistically identical. There was no statistically significant difference in haematocrit $(38.8 \pm 2.34$ vs $37.27 \pm 2.42 \%, p=0.3964)$, bleeding time $(4.24 \pm 0.48$ vs $4.24 \pm 0.5 \mathrm{~min}, \mathrm{p}=0.965)$ and clotting time $(5.89 \pm 0.42$ vs $5.88 \pm 0.41 \mathrm{~min}, p=0.958)$.

In pre-operative echocardiographic study left ventricular ejection fraction were studied in all patients. LVEF was divided into two groups. One was more than $50 \%$ and other was less than $50 \%$. In group A more than $18(75 \%)$ of the patient had LVEF $<50$ and $6(25 \%)$ had LVEF $>50$. In GroupB $17(70.8 \%)$ patient had LVEF $<50$ and 7 (29.2\%) patient had LVEF $>50$. But there was no significant difference in LVEF among the groups.

Pre-operative angiogram shows that there was also no significant difference between the two groups, most of the patients have triple vessel disease, $16(66.66 \%)$ in Group A and 15 (62.5\%) in Group - B.

Regarding per operative variables Group A and Group B total operation time is $5.89 \pm 0.25$ vs $5.01 \pm 0.37$ hours respectively and have no significant difference between the groups $(p>0.05)$. As heparin used at the beginning of the operation were neutralized at the end of operation by protamine so they are identical in both groups. Total operation time is standard for our country.

Difference between the two groups with regard to number of graft also not significant but most of the patients given three grafts (Group A $75.06 \%$ vs Group B $84.33 \%$ respectively). Xiao and his colleagues shows the mean number of distal anastomoses were $3.3 \pm 0.8$ in the patients group who discontinued aspirin for more than 5 days before 
surgery versus $3.2 \pm 0.8$ in the continued aspirin therapy group $(p=0.37)$. These are similar to our study. ${ }^{14}$

There is no significant difference between 2 groups with regards to activated clotting time, most of the reversal done at $100-120$ seconds, $91.6 \%$ and $87.5 \%$ in Group A and Group $-B$ respectively.

Our study shows post-operative blood losses up to removal of drain tubes was $643.33 \pm 36.67 \mathrm{ml}$ vs $648.33 \pm 37.6 \mathrm{ml}$; $\mathrm{p}=.643$. In our study there was no significant difference between the two groups with regard to blood loss. Most of patients needed 1 unit blood transfusion in both group (Group A $83.4 \%$ vs Group B $87.57 \%$ ) and also no patient required reoperation for bleeding.

Xiao and his colleagues shows there were no significant differences between preoperative non aspirin and aspirin therapy group with regard to postoperative blood loss (790 $\mathrm{ml}$ versus $800 \mathrm{ml}, \mathrm{p}=0.60$ ). Although not statistically significant, the rate for reoperation for bleeding was higher in aspirin users group ( $1.3 \%$ versus $2.4 \%, p=0.11)$. There were no significant differences among the two groups in blood transfusion rate $(24.4 \%$ versus $25.1 \%, p=0.76)$ and transfusion requirements of red blood cells, platelets and fresh frozen plasma. ${ }^{14}$

Post-operative ECG changes shows there was no significant difference between the two groups. Most of patients have normal ECG Group A $91.74 \%$ vs Group B $95.91 \%$ respectively. ST elevation appears in 2 (8.33\%) patients in Group A and 1 (4.16\%) patients in Group B. Q wave appears in 2 (8.33\%) patients in Group A and 1 (4.16\%) patients in Group B. Although not statistically significant but little bit higher rate of ST elevation and $Q$ wave found in Group -A (non aspirin group). There was no significant difference between the two groups with regard to new arrhythmia (Group A $25.02 \%$ \& Group B $20.85 \%$ ). Postoperative trop I level difference was also non-significant between 2 groups.

There was no significant difference between the two groups with regard to ICU stay. But most of the patients stayed 4 days, Group A $83.4 \%$ vs Group B $79.23 \%$ respectively.

Hospital stay was also not significant between the two groups. But most of patients released less than 10 days (Group A $70.89 \%$ vs Group B 75.06\%).

There was no significant difference between the two groups with regard to 30 days mortality. About 1 patients died within 30 days in Group-A. No mortality in Group B aspirin users. There was no incidence of thromboembolism, DVT or CVA in none of the patients in either group. Xiao and his colleagues shows there were no significant differences between preoperative nonaspirin and aspirin therapy group with regard to in-hospital mortality $(0.1 \%$ versus $0.1 \%, p=1.00)$, stroke $(0.1 \%$ versus $0.3 \%, p=1.00)$ and other thromboembolic manifestation. ${ }^{14}$

Bybee and his colleagues shows thirty-six of the total 1636 patients $(2.2 \%)$ died in-hospital after coronary bypass surgery. Of the 1316 patients receiving preoperative aspirin therapy, 22 died in-hospital after surgery whereas 14 of the 320 patients not receiving preoperative aspirin therapy died in-hospital after surgery. This resulted in an observed $61 \%$ relative reduction in all-cause in-hospital mortality in patients receiving preoperative aspirin therapy $(1.7 \%$ versus $4.4 \%)$ with a univariate $\mathrm{OR}$ of $0.37\left(95 \% \mathrm{Cl}, 0.19\right.$ to $\left.0.74 ; P \_0.004\right)$ for mortality). Patients receiving preoperative aspirin were less likely to die of cardiovascular causes $(0.5 \%)$ compared with those not receiving preoperative aspirin (2.2\%). There was no increased risk of reoperation for bleeding in those receiving preoperative aspirin therapy (3.5\% versus $3.4 \%$; OR, $1.02 ; 95 \% \mathrm{Cl}, 0.52$ to $\left.1.99 ; P \_0.96\right)$. There was a trend toward an increased need for postoperative blood product transfusion in the aspirin group, which did not reach statistical significance (OR, $1.25 ; 95 \% \mathrm{Cl}, 0.98$ to $\left.1.60 ; P \_0.07\right)$. There was no significant difference in the rates of postoperative adverse cerebrovascular events in those receiving preoperative aspirin compared with those not receiving preoperative aspirin by univariate analysis $(2.7 \%$ versus 3.8\%; OR, 0.72; $95 \% \mathrm{Cl}, 0.37$ to $\left.1.40 ; P \_0.34\right) .{ }^{10}$

Multiple studies support the safety of low-dose aspirin continuation in the context of cardiac surgery. Tuman and his colleagues compared perioperative use of aspirin versus placebo in patients undergoing reoperation coronary artery bypass graft (CABG). Of 317 total patients, 215 patients had taken aspirin within 7 days of their procedure versus none in their 102 matched controls. They found no significant differences in postoperative hematocrit, mediastinal drainage, the need for reoperation, or transfusion requirements. ${ }^{12}$

Srinivasan and his colleagues retrospectively examined 170 aspirin users presenting for first time off-pump coronary artery bypass compared to 170 matched controls, using propensity matching. They found no differences in mean postoperative blood loss ( $845 \mathrm{~mL}$ vs $775 \mathrm{~mL}, P=0.157$ ), the rate of reoperation for bleeding ( $3.5 \%$ vs $3.5 \%, P>0.99)$, blood product requirements, or in-hospital mortality. ${ }^{13}$

Sun and his colleagues published a review of the mixed evidence surrounding the risks and benefits of aspirin continuation up to the time of CABG surgery. They reported on 6 prospective studies that showed increased bleeding tendency with perioperative aspirin use, compared to 9 studies of varying methodologies (retrospective and 
prospective) indicating that perioperative aspirin did not increase transfusion needs. Although the authors do not make a definitive conclusion, they summarize their article by stating that overall, the bleeding risk posed to a patient by continuing on low-dose aspirin ( $<325 \mathrm{mg}$ ) for CABG surgery is likely to be less serious than the risk of a thromboembolic event.

\section{Conclusion:}

Preoperatively continued aspirin use was not associated with increased risk of post-operative blood loss, blood transfusion requirements, reoperation for bleeding and composite outcome of in hospital death and stroke in OPCAB. Thus, on the basis of the present study, we recommend that preoperative aspirin therapy should be continued till OPCAB surgery without interruption.

\section{References:}

1. Kaushansky K. Lineage-specific hematopoietic growth factors. N Engl J Med 2006; 354: 2034-45.

2. Cattaneo M. Antiplatelet agents. Hematology 2004; 5(3): 170-4.

3. Hennekens $\mathrm{CH}$, Dyken ML and Fuster V. Aspirin as a therapeutic agent in cardiovascular disease: a statement for healthcare professionals from the American Heart Association. Circulation 1997; 96: 2751-53.

4. Hall RI, MacLaren C, MclntyreAJ and Smith MS. Light versus heavy sedation after cardiac surgery: myocardial ischemia and the stress response Maritime Heart Centre and Dalhousie University. Anesth Analg J 1997; 85: 978-81.

5. Larsson PT, Wallen $\mathrm{NH}$ and Hjemdahl P. Norepinephrine-induced human platelet activation in vivo is only partly counteracted by aspirin. Circulation 1994; 89: 1951-7.

6. Vial JH, McLeod LJ and Roberts MS. Rebound elevation in urinary thromboxane B2 and 6-keto-PGF1 alpha excretion after aspirin withdrawal. Adv Prostagrandin T Thromboxane Leukot Res. 1991; 21: 157-60.

7. Václavík J and Táborský M. Antiplatelet therapy in the perioperative period. Eur J Intern Med 2011; 22: 26-31.

8. Wijns W, Kolh P, Folliguet T and Danchin N. Guidelines on myocardial revascularization: the Task Force on
Myocardial Revascularization of the European Society of Cardiology (ESC) and the European Association for Cardio-Thoracic Surgery. Eur Heart J 2010; 31: 2501-55.

9. Hillis LD, Smith PK, Hollar D and Anderson JL. ACCF/ AHA Guideline for Coronary Artery Bypass Graft Surgery, A report of the American College of Cardiology Foundation/ American Heart Association Task Force on Practice Guidelines. Developed in collaboration with the American Association for Thoracic Surgery, Society of Cardiovascular Anesthesiologists, and Society of Thoracic Surgeons. J Am Coll Cardiol 2011; 58(24): 123-210.

10. Bybee KA, Powell BD, Rosales G, Kopecky SL and Valeti U. Preoperative aspirin therapy is associated with improved postoperative outcomes in patients undergoing coronary artery bypass grafting. Circulation 2005; 112: 1286-90.

11. Chesebro JH, Fuster V, Clements IP, Smith HC and Elveback LR. Effect of dipyridamole and aspirin on late vein-graft patency after coronary bypass operations. N Engl J Med 1984; 310: 209-14.

12. Tuman KJ, McCarthy RJ, McCarthy WE and O'Connor CJ. Aspirin does not increase allogenic blood transfusion in reoperative coronary artery surgery. Anesth Analg J 1996; 83: 1178-84.

13. Srinivasan AK, Grayson AD, Fabri BM and Pullan DM. Effect of preoperative aspirin use in off-pump coronary artery bypass operations. Ann Thorac Surg 2003; 76 : $41-5$.

14. Ahsan KZ, Alam N, Pignone M, Albert MJ and Kimstreatfield P. Epidemiological transition in rural Bangladesh 1986-2006. Global Health Action 2009; 2: 1-23.

15. Xiao F, Wu H, Sun H, Pan S, Xu J and Song Y. Effect of Preoperatively Continued Aspirin Use on Early and Mid-Term Outcomes in Off-Pump Coronary Bypass Surgery: A Propensity Score-Matched Study of 1418 Patients. PLoS ONE 2015; 10(2): e0116311.

16. Sun JC, Whitlock R, Cheng J, Eikelboom JW and Thabane L. The effect of pre-operative aspirin on bleeding, transfusion, myocardial infarction, and mortality in coronary artery bypass surgery: A systematic review of randomized and observational studies. Eur Heart J 2008; 29: 1057-71. 